\title{
INTERSECTIONS OF CONTINUOUS, LIPSCHITZ, HÖLDER CLASS, AND SMOOTH FUNCTIONS
}

\author{
JACK B. BROWN
}

(Communicated by Andrew M. Bruckner)

\begin{abstract}
We present some improvements of known theorems and examples concerning intersections of continuous or Lipschitz functions with smooth functions or intersections of smooth functions or Hölder class functions with smoother functions. We are particularly concerned with our ability to force the projection of the intersection to be uncountable within a given set $M$ which is either large in measure or in category (or both).
\end{abstract}

\section{INTRODUCTION}

We shall restrict ourselves to the consideration of functions $f:[0,1] \rightarrow$ $\mathbb{R}$ which belong to the function classes indicated in the following diagram of implications:

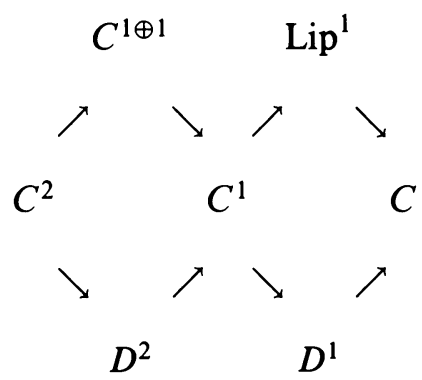

$C$ will denote the class of continuous functions and $C^{1}$ the class of continuously differentiable functions. Lip $^{1}$ will denote the class of functions such that $\{|f(x)-f(y)| /|x-y|: x, y \in[0,1]\}$ is bounded, and $C^{1 \oplus 1}$ denotes the "Hölder class" of $C^{1}$ functions $f$ such that $f^{\prime} \in \operatorname{Lip}^{1}$. For each $n \in \mathbb{N}, D^{n}$ denotes the class of $n$-times differentiable functions and $C^{n}$ denotes the class of $n$-times continuously differentiable functions. $\mathscr{L}$ denotes the Lebesgue measurable subsets of $[0,1]$, and $\mathscr{L}_{0}$ denotes the measure zero sets in $\mathscr{L}$. Thus, $\mathscr{L} \backslash \mathscr{L}_{0}$ denotes the sets of positive measure and co- $\mathscr{L}_{0}$ denotes the sets of full

Received by the editors February 18, 1993 and, in revised form, July 8, 1993; these results were presented at the 16th Summer Symposium on Real Analysis, held in Wolfville, Nova Scotia, in August, 1992.

1991 Mathematics Subject Classification. Primary 26A16; Secondary 26A15.

Key words and phrases. Differentiable, Lipschitz, Hölder, smooth functions. 
measure. The statement that a subset $M$ of $[0,1]$ is measure dense in a subinterval $I$ of $[0,1]$ means that $\lambda(M \cap J)>0$ for every subinterval $J$ of $I . B_{\mathrm{w}}$ denotes the sets with the Baire property (wide sense) [4], FC denotes the first category sets, and co-FC denotes the residual sets.

The first theorem of the type mentioned in the abstract which we consider is the following theorem due to Laczkovich [5] and Agronski, Bruckner, Laczkovich, and Preiss [1].

Theorem 1. If $M \in \mathscr{L} \backslash \mathscr{L}_{0}$, then for every $f \in C$ there exists a $g \in C^{1}$ such that $\{x \in M: f(x)=g(x)\}$ is uncountable.

Thus, continuous functions have uncountable intersections with $C^{1}$ functions and you can make the projection of this intersection be uncountable inside any given set of positive measure. On the other hand, it is fairly easy to see that you cannot necessarily make this intersection be uncountable inside any given $B_{w} \backslash F C$ set because of the following partial converse to Theorem 1.

Theorem 2. If $M \in \mathscr{L}_{0}$, there is an $f \in C$ such that $\{x \in M: f(x)=g(x)\}$ is countable for every $g \in D^{1} \cup \mathrm{Lip}^{1}$.

Proof. Let $M \in \mathscr{L}_{0}$. It follows that there is a sequence $C_{1}, C_{2}, \ldots$ of pairwise disjoint Cantor subsets of $[0,1]$ such that $\lambda\left(C_{1} \cup C_{2} \cup \cdots\right)=1$ and $M \subseteq G=$ $[0,1] \backslash\left(C_{1} \cup C_{2} \cup \cdots\right)$. The $C_{i}$ can be chosen so that $1 \lambda\left(C_{1}\right)+2 \lambda\left(C_{2}\right)+3 \lambda\left(C_{3}\right)+$ ‥ converges. Let $h:[0,1] \rightarrow \mathbb{R}$ be such that $h(x)=i$ if $x \in C_{i}$ for some $i \in \mathbb{N}$, and $h(x)=0$ otherwise. Define $f(x)=\int_{0}^{x} h(t) d t$. It follows that $f$ is differentiable in the "extended sense" on $G$, with $f^{\prime}(x)=+\infty$ for every $x \in G$. Thus, $\{x \in M: f(x)=g(x)\}$ is countable for every $g \in D^{1} \cup$ Lip $^{1}$.

\section{INTERSECTION OF LIPSCHITZ, HÖLDER CLASS, AND SMOOTH FUNCTIONS}

In 1944 Federer [2] (also see [3, Theorem 3.1.15]) proved a theorem from which it follows that functions in $D^{1} \cup \mathrm{Lip}^{1}$ intersect $C^{1}$ functions and that you can make the projection of the intersection be of positive measure inside any given $\mathscr{L} \backslash \mathscr{L}_{0}$ set $M$. We now prove a category version of this result.

Theorem 3. If $M \in B_{w} \backslash F C$, then for every $f \in D^{1} \cup$ Lip $^{1}$ there exists a $g \in C^{1}$ such that $\{x \in M: f(x)=g(x)\}$ is uncountable.

Proof. $M$ will be residual in some subinterval of $[0,1]$. We assume without loss of generality that $M$ is co-FC on $[0,1]$ and let $G_{1} \supseteq G_{2} \supseteq \cdots$ be a sequence of dense open subsets of $[0,1]$ such that $G_{1} \cap G_{2} \cap \cdots \subseteq M$.

We first consider the case where $f \in \operatorname{Lip}^{1}$ (the $D^{1}$ case follows easily from this case). We know that $f$ is a.e. differentiable, that $f^{\prime}$ is bounded, and that $f(x)=\int_{0}^{x} f^{\prime}(t) d t$ for every $x \in[0,1]$. Let $M$ and $m$ be the supremum and infimum, respectively, of the range of $f^{\prime}$. We assume without loss of generality that $0 \leq m<M \leq 1$. Otherwise, we could work with the function $F(x)=\int_{0}^{x} F^{\prime}(t) d t$, where $F^{\prime}(x)=\left(f^{\prime}(x)-m\right) /(M-m)$, and transform back to $f$ at the end of the argument.

Case $\mathrm{I}$. We assume there is a number $0<t<1$ and an interval $I=[a, b] \subseteq$ $[0,1]$ such that the sets $U_{t}=\left\{x: f^{\prime}(x)>t\right\}$ and $V_{t}=\left\{x: f^{\prime}(x)<t\right\}$ are both measure dense in $I$. It follows that the function $h(x)=f(x)-f(a)-t(x-a)$ is "nowhere monotone" on $I$ (i.e., monotone on no subinterval of $I$ ). It follows 
[6] that there is a number $s$ such that the set $T=\{x \in I: h(x)=s\}$ is uncountable. Let $x_{0}$ and $x_{1}$ be elements of $T$. Using continuity of $h$, we can find short pairwise disjoint closed intervals $I_{0}$ and $I_{1}$ located close to $x_{0}$ and $x_{1}$, respectively, lying interior to $G_{1} \cap I$, having common length $d_{1}$, and such that if $x \in I_{0}$ and $y \in I_{1}$, then $|x-y|>d_{1}$ and $|h(y)-h(x)| /|y-x|<1 / 1$. This completes stage 1 of the construction.

Now, suppose $n$ is a positive integer and that the intervals $\left\{I_{b}: b \in\{0,1\}^{n}\right\}$, all having common length $d_{n}$, have been constructed. Let $b \in\{0,1\}^{n}$. There is a number $s_{b}$ such that the set $T_{b}=\left\{x \in \dot{I}_{b}: h(x)=s_{b}\right\}$ is uncountable. Let $x_{b 0}$ and $x_{b 1}$ be two elements of $T_{b}$. We can find short pairwise disjoint closed intervals $I_{b 0}$ and $I_{b 1}$ close to $x_{b 0}$ and $x_{b 1}$, respectively, lying interior to $G_{n+1} \cap I_{b}$, having length $d_{n+1}<d_{n} / 2$ (same $d_{n+1}$ for all $b \in\{0,1\}^{n}$ ), and such that if $x \in I_{b 0}$ and $y \in I_{b 1}$, then $|x-y|>d_{n+1}$ and $|h(y)-h(x)| /|y-x|<$ $1 /(n+1)$.

Now, let $P=\bigcap_{n \in \mathbb{N}} \cup\left\{I_{b}: b \in\{0,1\}^{n}\right\} . P$ is a perfect subset of $G_{1} \cap G_{2} \cap$ $\cdots \subseteq M$ and $h \mid P$ satisfies the "uniform differentiability" requirements of the Whitney Extension Theorem [8; 3, Theorem 3.1.14] which guarantee that $h \mid P$ can be extended to a $C^{1}$ function $H$. Then, the $C^{1}$ function $g$ defined by $g(x)=H(x)+f(a)+t(x-a)$ agrees with $f$ over $P$, and the theorem is proved in this case.

Case II. We assume the bare denial of Case I (i.e., for every number $0<t<1$ and every subinterval $I$ of $[0,1]$, there is a subinterval $J$ of $I$ such that either $\lambda\left(U_{t} \cap J\right)=0$ or $\left.\lambda\left(V_{t} \cap J\right)=0\right)$. Let $n \in \mathbb{N}$. It follows from the assumptions that there will exist an interval $I \subseteq[0,1]$ and an integer $i, 1 \leq i \leq n$, such that $\lambda\left(\left\{x \in I: f^{\prime}(x)>i / n\right.\right.$ or $\left.\left.f^{\prime}(x)<(i-1) / n\right\}\right)=0$. To see this, first start with $i=n$ and $t=i / n$. There is a subinterval $J_{n}$ of $[0,1]$ such that either $\lambda\left(U_{t} \cap J_{n}\right)=0$ or $\lambda\left(V_{t} \cap J_{n}\right)=0$. If it is the latter case set $I=J_{n}$ and we are through. Otherwise, set $i=n-1$ and repeat to get $J_{n-1} \subseteq J_{n}$. If $\lambda\left(V_{t} \cap J_{n-1}\right)=0$, we are through. Otherwise, if necessary, continue the process until we get to $J_{1} \subseteq J_{2}$, which must suffice for $I$.

Consequently, there will exist a collection $H_{n}$ of such intervals $I$ such that the intervals in $H_{n}$ are pairwise disjoint, the closure of each interval in $H_{n}$ lies in the interior of an interval of $H_{n-1}$, and $\bigcup H_{n}$ is dense in $[0,1]$. Let $H=\bigcap_{n \in \mathbb{N}} \cup H_{n}$.

It follows that for every $x \in H, f^{\prime}(x)$ exists. To see this, let $I_{1}, I_{2}, \ldots$ be the sequence of intervals from $H_{1}, H_{2}, \ldots$, respectively, containing $x$. For each $n$, let $i_{n}$ be the integer $i$ described above for which

$$
\lambda\left(\left\{y \in I_{n}: f^{\prime}(y)>i / n \text { or } f^{\prime}(y)<(i-1) / n\right\}\right)=0 .
$$

Notice that the sequences $\left\{\left(i_{n}-1\right) / n\right\}$ and $\left\{i_{n} / n\right\}$ both converge to some common number $m$. $f^{\prime}(x)$ must exist and equal $m$. To see this suppose $\varepsilon>0$ and let $n$ be such that $1 / n<\varepsilon$. Consider any $y$ in $I_{n}$. [ $\left.f(y)-f(x)\right] /(y-x)=$ $\int_{x}^{y} f^{\prime}(t) d t /(y-x)$, which lies between $\left(i_{n}-1\right) / n$ and $i_{n} / n$, which in turn differ by less than $\varepsilon$ and also have $m$ between them.

It is clear that for every $x \in H, f^{\prime}$ is "essentially continuous" at $x$ (i.e., for every $\varepsilon>0$, there exists $\delta>0$ such that $\left\{y:|x-y|<\delta, f^{\prime}(y)\right.$ exists, and $\left.\left|f^{\prime}(y)-f^{\prime}(x)\right| \geq \varepsilon\right\}$ is $\left.\mathscr{L}_{0}\right)$.

We will now describe a special Cantor subset $P$ of $G=G_{1} \cap G_{2} \cap \ldots$ (defined at beginning of proof). Choose two points $x_{0}$ and $x_{1}$ of $G \cap H$ and two 
disjoint closed neighborhoods $I_{0}$ and $I_{1}$ of $x_{0}$ and $x_{1}$, respectively, lying in $G_{1}$, lying inside intervals of $H_{1}$, and having length $<1 / 2$ and two open intervals $J_{0}$ and $J_{1}$ of length less than $1 / 2$ such that $\lambda\left(\left\{x \in I_{i}: f^{\prime}(x)\right.\right.$ exists and $\left.\left.f^{\prime}(x) \notin J_{i}\right\}\right)=0$ for $i=0,1$. Let $K$ be the closed interval lying between $I_{0}$ and $I_{1}$.

Now suppose $n$ is a positive integer and $b \in\{0,1\}^{n}$. Choose two points $x_{b 0}$ and $x_{b 1}$ of $G \cap H$ interior to $I_{b}$ and two disjoint closed neighborhoods $I_{b 0}$ and $I_{b 1}$ of $x_{b 0}$ and $x_{b 1}$, respectively, lying in $G_{n+1} \cap I_{b}$, lying inside intervals of $H_{n+1}$, and having length $<1 / 2^{n+1}$ and two open intervals $J_{b 0}$ and $J_{b 1}$ of length less than $1 / 2^{n+1}$, lying inside $J_{b}$, and such that

$$
\lambda\left(\left\{x \in I_{b i}: f^{\prime}(x) \text { exists and } f^{\prime}(x) \notin J_{b i}\right\}\right)=0
$$

for $i=0,1$. Let $K_{b}$ be the closed interval lying between $I_{b 0}$ and $I_{b 1}$.

The set $P=\bigcap_{n \in \mathbb{N}} \cup\left\{I_{b}: b \in\{0,1\}^{n}\right\}$ is a perfect subset of $G$ and $f^{\prime} \mid P$ is continuous. We now extend $f^{\prime} \mid P$ in a special way to a continuous function $h$ on $[0,1]$. First let $(c, d)$ be the interval contiguous to $P$ which contains the interval $K$. Notice that $f^{\prime}(x) \in(-0.5,1.5)$ for a.e. $x \in(c, d)$, so $h$ can be defined continuously on $[c, d]$ so that $(1) h(c)=f^{\prime}(c)$ and $h(d)=f^{\prime}(d),(2)$ $h(x) \in(-0.5,1.5)$ for every $x \in(c, d)$, and $(3) \int_{c}^{d} h(t) d t=\int_{c}^{d} f^{\prime}(t) d t$. This can be accomplished in two steps. First choose $c^{\prime}$ close to $c$ and $d^{\prime}$ close to $d$ with $c<c^{\prime}<d^{\prime}<d$. Let $h_{0}(x)=f^{\prime}(c)$ on $\left[c, c^{\prime}\right)$ and $h_{0}(x)=f^{\prime}(d)$ on $\left(d^{\prime}, d\right]$. For $c^{\prime}-c$ and $d-d^{\prime}$ small enough we can set $h_{0}(x)$ equal to some constant $k \in(-0.5,1.5)$ for $x \in\left[c^{\prime}, d^{\prime}\right]$ so that (3) holds for $h_{0}$ in place of $h$. Then for some small $\delta>0$, we can let $h(x)=h_{0}(x)$ on $\left[c, c^{\prime}-\delta\right]$, $\left[c^{\prime}+\delta, d^{\prime}-\delta\right]$, and $\left[d^{\prime}+\delta, d\right]$, and let $h$ be linear across the gaps $\left[c^{\prime}-\delta, c^{\prime}+\delta\right]$ and $\left[d^{\prime}-\delta, d^{\prime}+\delta\right]$, and we will have the desired $h$.

Suppose $n$ is a positive integer and $b \in\{0,1\}^{n}$. Let $(c(b), d(b))$ be the interval contiguous to $P$ which contains the interval $K_{b}$. Notice that $f^{\prime}(x) \in J_{b}$ for a.e. $x \in(c(b), d(b))$; so using the method described above, $h$ can be defined continuously on $[c(b), d(b)]$ so that $(1) h(c(b))=f^{\prime}(c(b))$ and $h(d(b))=f^{\prime}(d(b)),(2) h(x) \in J_{b}$ for every $x \in(c(b), d(b))$, and (3) $\int_{c(b)}^{d(b)} h(t) d t=\int_{c(b)}^{d(b)} f^{\prime}(t) d t$.

Set $h\left|P=f^{\prime}\right| P$, and extend $h$ continuously to the left and right of $P$. Let $a$ be the least element of $P$. Now, if $g$ is defined by $g(x)=f(a)+\int_{a}^{x} h(t) d t$, it follows that $f|P=g| P$, and this completes the proof for the case $f \in \operatorname{Lip}^{1}$.

If $f \in D^{1}$, then there exists an interval $I \subseteq[0,1]$ relative to which $f$ is $\mathrm{Lip}^{1}$, and the argument given above will apply relative to $I$.

Remark. Federer's original theorem [2] does not just apply to functions in $D^{1} \cap \operatorname{Lip}^{1}$, it actually yields the desired conclusion for functions which are continuous and which are what would be called "a.e. pointwise $\mathrm{Lip}^{1}$ ". This class of functions is much larger than $D^{1} \cup \operatorname{Lip}^{1}$ and, in fact, includes the absolutely continuous (and even the CBV) functions. We did not state our Theorem 3 for this larger class because the conclusion of our Theorem 3 does not even hold for the absolutely continuous functions. The function constructed in the proof of Theorem 2 shows this.

Of course, we cannot get the exact category analog of Federer's intersection 
theorem, obtaining $\{x \in M: f(x)=g(x)\} \in \mathrm{B}_{\mathrm{w}} \backslash \mathrm{FC}$ at the end, because of the following.

Example 4. There exists a $\operatorname{Lip}^{1}$ function $f$ such that for every $D^{1} g$, the set $\{x: f(x)=g(x)\}$ is nowhere dense.

Proof. Let $C_{1}, C_{2}, \ldots$ be a sequence of pairwise disjoint Cantor Subsets of $[0,1]$ such that $\lambda\left(C_{1} \cup C_{2} \cup \cdots\right)=1$ and such that if $x \in C_{n}$ for some $n$, then $C_{n}$ has one-sided (left or right) upper density equal to 1 at $x$. Let $M=$ $[0,1] \backslash\left(C_{1} \cup C_{2} \cup \cdots\right)$. Define $h$ so that $h(x)=1 / n$ if $x \in C_{n}$ and $h(x)=0$, otherwise. Let $f(x)=\int_{0}^{x} h(t) d t$. It is easily seen that $f$ is differentiable on $M$ and that $f^{\prime}(x)=0$ for every $x \in M$; and it follows from the density requirements on the $C_{n}$ that if $f$ is differentiable at some $x \in C_{n}$, then $f^{\prime}(x)=1 / n$. Suppose $g$ is a $D^{1}$ function such that the set $A=\{x: f(x)=$ $g(x)\}$ is dense in some interval $I$. Since $A$ is closed, it follows that $f(x)=$ $g(x)$ for every $x \in I$. This would imply that the range of $g^{\prime} \mid I$ is a disconnected set, which is a contradiction.

In proving Theorems 6 and 9, we will need the following lemma, which was essentially proved by Olevskii [7] without the extra stipulations involving the set $M$.

Lemma 5. If $M \in B_{w} \backslash F C, f \in C^{1}$, and $f^{\prime}$ is nowhere monotone, then there exists a $g \in C^{2}$ such that $\{x \in M: f(x)=g(x)\}$ is uncountable.

Proof. We will make some slight modifications of Olevskii's proof of Theorem 2 of [7] to account for the set $M$ (we will also be assuming Olevskii's Lemmas 2 and 3). If $M \in \mathrm{B}_{\mathrm{w}} \backslash \mathrm{FC}, M$ is residual in some interval $I$ (assume without loss of generality that $I=[0,1])$, and there will exist a sequence $G_{1} \supseteq G_{2} \supseteq \cdots$ of dense open subsets of $I$ such that $G_{1} \cap G_{2} \cap \cdots \subseteq M . f^{\prime}$ is nowhere monotone, so pick the points $a_{1}$ and $a_{2}$ in Olevskii's argument for which $f^{\prime}\left(a_{1}\right)=f^{\prime}\left(a_{2}\right)=\left[f\left(a_{2}\right)-f\left(a_{1}\right)\right] /\left(a_{2}-a_{1}\right)$ to lie inside a common component of $G_{1}$. Then, pick the $\delta_{i} \quad(i=1,2)$ in his argument small enough so that the closed neighborhoods $\Delta_{i}(i=1,2)$ lie interior to $G_{1}$, in addition to satisfying Olevskii's other requirements. Likewise, pick the points $a_{i j}(i, j=1,2)$ and the $\delta_{2}$ small enough that the closed neighborhoods $\Delta_{i j}(i, j=1,2)$ lie interior to $G_{2}$ and satisfy Olevskii's other requirements. Continuing this process, we will force Oleveskii's resulting set $E$ (on which $f$ agrees with some $g \in C^{2}$ ) to lie inside $G_{1} \cap G_{2} \cap \cdots \subseteq M$.

Olevskii [7] proved that $C^{1}$ functions necessarily have uncountable intersections with $C^{2}$ functions, but he did not address the question of making the projection of the intersection be uncountable within some given large set $M$. We do this in the following theorem.

Theorem 6. If $M \in B_{w} \cap \mathscr{L}$ and $M$ is measure dense in some interval $I$ in which $M$ is residual, then for every $f \in C^{1}$ there exists a $g \in C^{2}$ such that $\{x \in M: f(x)=g(x)\}$ is uncountable.

Proof. If there exists an interval $B \subseteq I$ on which $f^{\prime}$ is monotone, then $f^{\prime \prime}$ exists a.e. on $B$ and there will exist a set $A \subseteq B \cap M, A \in \mathscr{L} \backslash \mathscr{L}_{0}$, such that $f^{\prime \prime} \mid A$ is bounded. It follows from [3, Theorem 3.1.15] that there is a $C^{2} \quad g$ such that $\{x \in A: f(x)=g(x)\}$ is of positive measure and therefore uncountable. 
If, on the other hand, $f^{\prime}$ is nowhere monotone on $I$, the result follows from Lemma 5.

The requirements on $M$ in the hypothesis of Theorem 6 seem to be quite strong at first glance. Still, the author actually believes that the converse (for $M \in \mathrm{B}_{\mathrm{w}} \cap \mathscr{L}$ ) of the theorem holds, but he has been unable to prove this. In any case, the following two examples show that weaker requirements would be insufficient.

Example 7. There exists an $F_{\sigma}$, co- $\mathscr{L}_{0}$ set $M$ and an $F \in C^{1}$ such that for every $g \in D^{2} \cup C^{1 \oplus 1},\{x \in M: F(x)=g(x)\}$ is countable.

Proof. Let $P_{1}$ be the Cantor set $P, h_{1}$ be the function $h$, and $f_{1}$ be the function $f$ constructed in the proof of Theorem 22 (with $\varepsilon=0.5$ ) of [1]. If $[a, b]$ is a subinterval of $[0,1]$, let

$$
P_{[a, b]}=\left\{x: x=a+(b-a) y \text { for some } y \in P_{1}\right\},
$$

and let $h_{[a, b]}$ be the function defined by

$$
h_{[a, b]}(x)=(b-a) h_{1}((x-a) /(b-a)) \text { for each } x \in[a, b] \text {. }
$$

Let $P_{2}=\bigcup\left\{P_{[a, b]}:(a, b)\right.$ is an interval contiguous to $\left.P_{1}\right\} \cup P_{1}$, let $h_{2}$ be the function such that $h_{2}(x)=h_{[a, b]}(x)$ if $x$ is in an interval $(a, b)$ contiguous to $P_{1}$ and $h_{2}(x)=0$ if $x \in P_{1}$, and define $f_{2}(x)=\int_{0}^{x} h_{2}(t) d t$. For each $n$, let $P_{n}=\bigcup\left\{P_{[a, b]}:(a, b)\right.$ is an interval contiguous to $\left.P_{n-1}\right\} \cup P_{n-1}$, let $h_{n}$ be the function such that $h_{n}(x)=h_{[a, b]}(x)$ if $x$ is in an interval $(a, b)$ contiguous to $P_{n-1}$, let $h_{n}(x)=0$ if $x \in P_{n-1}$, and define $f_{n}(x)=\int_{0}^{x} h_{n}(t) d t$. Each $h_{n}$ is continuous, and $h_{1}+h_{2}+\cdots$ converges uniformly to a continuous function that we denote by $H$. We denote the $C^{1}$ limit of $f_{1}+f_{2}+\cdots$ by $F$. Let $M=P_{1} \cup P_{2} \cup \cdots$, which is co- $\mathscr{L}_{0}$.

Suppose there is a $g \in D^{2} \cup C^{1 \oplus 1}$ such that $M^{\prime}=\{x \in M: F(x)=g(x)\}$ is uncountable. Either $M^{\prime} \cap P_{1}$ is uncountable or there is a first $n \geq 2$ such that $M^{\prime} \cap P_{[a, b]}$ is uncountable for some interval $(a, b)$ contiguous to $P_{n-1}$. If $M^{\prime} \cap P_{1}$ is uncountable, we can achieve a contradiction similar to the contradiction obtained in [1] and similar to the contradiction we obtain in the more complicated latter case, so we assume the latter is the case. It follows that there is a decreasing sequence $x_{1}, x_{2}, \ldots$ of elements of $M^{\prime} \cap P_{[a, b]}$ converging to an element $x_{0}$ of $M^{\prime} \cap P_{[a, b]}$ such that each $x_{i}$ is a limit point of $M^{\prime}$. We may also assume that all of the $x_{i}$ lie in a neighborhood $N$ of $x_{0}$ which contains no midpoint of any interval $\left(a_{j}, b_{j}\right)$ containing $(a, b)$ and contiguous to $P_{j}$ for any $j \leq n-1$. It follows that each $h_{j}(j=1,2, \ldots, n-1)$ is continuously differentiable on $N$ and that $E_{n-1}=f_{1}+f_{2}+\cdots+f_{n-1}$ is twice continuously differentiable on $N$. Let $F_{n}=F-E_{n-1}, G=g-E_{n-1}$, and $H_{n}=h_{n}+h_{n+1}+\cdots$.

In the case that $g \in D^{2}$, it follows that $G$ is twice differentiable on $N$ and $G^{\prime}\left(x_{0}\right)=F_{n}^{\prime}\left(x_{0}\right)=H_{n}\left(x_{0}\right)=0$, so that L'Hôpital's rule can be applied as it was in [1] to conclude that

$$
\lim _{i \rightarrow \infty}\left[G\left(x_{i}\right)-G\left(x_{0}\right)\right] /\left(x_{i}-x_{0}\right)^{2}=G^{\prime \prime}\left(x_{0}\right) / 2 \text {. }
$$

On the other hand, $G\left(x_{i}\right)=F_{n}\left(x_{i}\right)$ for $i=0,1,2, \ldots$, and the geometry of the construction of $h_{[a, b]}$ is similar enough to that in [1] to conclude that since

$$
\lim _{i \rightarrow \infty}\left[f_{n}\left(x_{i}\right)-f_{n}\left(x_{0}\right)\right] /\left(x_{i}-x_{0}\right)^{2}=+\infty \text {, }
$$


the larger difference quotients will do the same and

$$
\lim _{i \rightarrow \infty}\left[F_{n}\left(x_{i}\right)-F_{n}\left(x_{0}\right)\right] /\left(x_{i}-x_{0}\right)^{2}=+\infty \text {. }
$$

This provides the desired contradiction for the case $g \in D^{2}$.

In case $g \in C^{1 \oplus 1}$, we still have that $G$ is differentiable on $N, G^{\prime}\left(x_{0}\right)=0$, and $G^{\prime}$ is $\operatorname{Lip}^{1}$ (relative to $N$ ). For each $i$, there will exist $x_{0}<y_{i}<x_{i}$ such that

$$
\left[G\left(x_{i}\right)-G\left(x_{0}\right)\right] /\left(x_{i}-x_{0}\right)^{2}=G^{\prime}\left(y_{i}\right) /\left(x_{i}-x_{0}\right)=\left[G^{\prime}\left(y_{i}\right)-G^{\prime}\left(x_{0}\right)\right] /\left(x_{i}-x_{0}\right)
$$

so that, since $G^{\prime}$ is $\operatorname{Lip}^{1}$ relative to $N$,

$$
\limsup _{i \rightarrow \infty}\left[G\left(x_{i}\right)-G\left(x_{0}\right)\right] /\left(x_{i}-x_{0}\right)^{2}<+\infty .
$$

But this contradicts the fact that

$$
\lim _{i \rightarrow \infty}\left[F_{n}\left(x_{i}\right)-F_{n}\left(x_{0}\right)\right] /\left(x_{i}-x_{0}\right)^{2}=+\infty,
$$

which still holds.

Example 8. There exists an $N \in\left(\mathscr{L} \backslash \mathscr{L}_{0}\right) \cap$ (co-FC) and an $f \in C^{1}$ such that $\{x \in M: f(x)=g(x)\}$ is countable for every $g \in D^{2} \cup C^{1 \oplus 1}$.

Proof. Again, let $\varepsilon=1 / 2, P_{1}$ be the set $P$ with $\lambda(P)=1-\varepsilon$, and $f_{1}$ be the function $f$ described in the proof of Theorem 22 of [1]. Let $P_{2}, P_{3}, \ldots$ be a sequence of pairwise disjoint Cantor subsets of $[0,1] \backslash P_{1}$ such that $\lambda\left(P_{i}\right)=1 / 2^{i}$ for $i=2,3, \ldots$. Let $k$ be the function $f$ described in Theorem 2 with $M=[0,1] \backslash\left(P_{1} \cup P_{2} \cup \cdots\right)$, and let $f_{2}(x)=\int_{0}^{x} k(t) d t$. The function $f=f_{1}+f_{2}$ is $C^{1}$ and $N=P_{1} \cup M$ is residual and of positive measure.

Suppose $g \in D^{2} \cup C^{1 \oplus 1}$ and that the set $A=\{x \in N: f(x)=g(x)\}$ is uncountable.

If $A \cap M$ is uncountable, then $A \cap M$ contains a condensation point $x$ of itself such that $x$ is not a midpoint of any interval contiguous to $P_{1}$. It follows that $f_{1}^{\prime \prime}(x)=0$ but $f_{2}^{\prime \prime}(x)=+\infty$, so that $f^{\prime \prime}(x)=D^{+} g(x)=+\infty$, which is a contradiction. $D^{+}$and $D_{+}$are the notation for the upper and lower right Dini derivatives.

If $A \cap P_{1}$ is uncountable, it follows that there is a convergent decreasing sequence $x_{k} \rightarrow x_{0}$, where $x_{k} \in A \cap P_{1}$, for $k=0,1,2, \ldots$, and each $x_{k}$ is a bilateral condensation point of $A \cap P_{1}$. It follows, as in [1], that $D^{+} f_{1}^{\prime}\left(x_{0}\right)=$ $+\infty$. Since $f_{2}^{\prime}=k$ is strictly increasing, $D_{+} f_{2}^{\prime}\left(x_{0}\right) \geq 0$. Thus, $D^{+} f^{\prime}\left(x_{0}\right)=$ $D^{+} g^{\prime}\left(x_{0}\right)=+\infty$, which is a contradiction.

In 1951 Whitney [9] (also see [3, Theorem 3.1.15]) proved a theorem from which it follows that functions in $D^{2} \cup C^{1 \oplus 1}$ intersect $C^{2}$ functions and that the projection of the intersection could be made of positive measure inside any given $\mathscr{L} \backslash \mathscr{L}_{0}$ set. We now prove the following category analog of that result.

Theorem 9. If $M \in B_{w} \backslash F C$, then for every $f \in D^{2} \cup C^{1 \oplus 1}$ there exists a $g \in C^{2}$ such that $\{x \in M: f(x)=g(x)\}$ is uncountable.

Proof. The proof is essentially similar to the proof of Theorem 3. We start with the same sets $G_{1}, G_{2}, \ldots$ and proceed by replacing $f$ in that proof with $f^{\prime}$ in this argument and replacing $f^{\prime}$ in that proof with $f^{\prime \prime}$ in this argument. We first assume $f \in C^{1 \oplus 1}$. 
Case I. Because the sets $U_{t}=\left\{x: f^{\prime \prime}(x)<t\right\}$ and $V_{t}=\left\{x: f^{\prime \prime}(t)>t\right\}$ are both measure dense in $I$, it follows that the function $h(x)=f^{\prime}(x)-f^{\prime}(a)-$ $t(x-a)$ is nowhere monotone on $I$. We then set

$$
H(x)=f(x)-f^{\prime}(a)(x-a)-t(x-a)^{2} / 2,
$$

call on Lemma 5 as it applies to the function $H$, and use this to finish the proof for this case.

Case II. We proceed as we did in the proof of Theorem 3, using $f^{\prime \prime}$ in place of $f^{\prime}$, obtaining the similar sets $H_{n}$ and the set $H$ on which $f^{\prime \prime}$ is essentially continuous. We define similar points $x_{b}$ and intervals $I_{b}, J_{b}$, and $K_{b}$ for finite binary sequences $b$, using $f^{\prime \prime}$ in place of $f^{\prime}$, obtaining a similar perfect set $P$. We then need to extend $f^{\prime \prime} \mid P$ to a continuous function $h$ on $[0,1]$, but there is an extra requirement on the extension in this argument. At the point where $b \in[0,1]^{n}$ and $(c(b), d(b))$ is the interval contiguous to $P$ which contains the interval $K_{b}$ we define $h$ continuously on $[c(b), d(b)]$ so that $(1) h(c(b))=f^{\prime \prime}(c(b))$ and $h(d(b))=f^{\prime \prime}(d(b))$, (2) $h(x) \in J_{b}$ for every $x \in(c(b), d(b))$, and (3) $h_{1}(d(b))-h_{1}(c(b))=f^{\prime}(d(b))-f^{\prime}(c(b))$ and (4) $h_{2}(d(b))-h_{2}(c(b))=f(d(b))-f(c(b))$, where $h_{1}(x)=\int_{c(b)}^{x} h(t) d t$ and $h_{2}(x)=\int_{c(b)}^{x} h_{1}(t) d t$.

Construction of this function $h$ is a bit more complicated than the construction of the function $h$ in Case II of the proof of Theorem 3. To illustrate the method of construction, assume $(c, d)$ is an interval such that $f^{\prime \prime}(c)$ and $f^{\prime \prime}(d)$ exist and lie in an interval $J=(u, v)$, which is such that $u<f^{\prime \prime}(x)<v$ for a.e. $x \in(c, d)$. Consider what the function $f^{\prime}$ must be like on $[c, d]$. Extend lines $U_{1}$ and $V_{1}$ with slopes $u$ and $v$, respectively, to the right from $\left(c, f^{\prime}(c)\right)$ and lines $U_{2}$ and $V_{2}$ with slopes $u$ and $v$, respectively, to the left from $\left(d, f^{\prime}(d)\right)$. Since $u<f^{\prime \prime}(x)<v$ a.e. on $(c, d)$ and $f^{\prime}(x)=$ $f^{\prime}(c)+\int_{c}^{x} f^{\prime \prime}(t) d t$ for $x \in[c, d]$, it follows that these four lines form a parallelogram $Q$ with $\left(c, f^{\prime}(c)\right.$ and $\left(d, f^{\prime}(d)\right)$ at opposite corners. It is also the case that $f^{\prime} \mid(c, d)$ lies interior to the parallelogram $Q$. Now, choose $c^{\prime}$ close to $c$ and $d^{\prime}$ close to $d$ with $c<c^{\prime}<d^{\prime}<d$. Let $h_{0}(x)=f^{\prime}(c)+f^{\prime \prime}(c)(x-c)$ for $x \in\left[c, c^{\prime}\right]$, and let $h_{0}(x)=f^{\prime}(d)+f^{\prime \prime}(d)(x-d)$ for $x \in\left[d^{\prime}, d\right]$. Assuming $c^{\prime}-c$ and $d-d^{\prime}$ are small enough, it will be possible to choose an $s \in\left(c^{\prime}, d^{\prime}\right)$ and extend $h_{0}$ continuously on $\left[c^{\prime}, d^{\prime}\right]$ to be linear on $\left[c^{\prime}, s\right]$ and linear on $\left[s, d^{\prime}\right]$, with the slopes of both extensions between $u$ and $v$, and in such a way that $\int_{c}^{d} h_{0}(t) d t=\int_{c}^{d} f^{\prime}(t) d t . h_{0}$ is polygonal line graph on $[c, d]$, and we now need to "round off" the corners which occur at $\left(c^{\prime}, h_{0}\left(c^{\prime}\right)\right)$, $\left(s, h_{0}(s)\right)$, and $\left(d^{\prime}, h_{0}\left(d^{\prime}\right)\right)$ to make it $C^{1}$. In this smoothing operation, we must make sure that the resulting function $h_{1}$ still satisfies $u<h_{1}^{\prime}(x)<v$ for $x \in[c, d]$ and $\int_{c}^{d} h_{1}(t) d t=\int_{c}^{d} f^{\prime}(t) d t$.

Now, we define $h_{1}$ like this on each interval $(c, d)=(c(b), d(b))$ for $b \in$ $\{0,1\}^{n}$ and $J=J_{b}$, as described in the previous paragraph, and define $h_{1} \mid P=$ $f^{\prime} \mid P$. Then $h_{1}^{\prime}$ is the desired function $h$.

Then, if $a$ is the least element of $P$ and $g$ is defined by $g(x)=f(a)+$ $\int_{a}^{x} g_{1}(t) d t$, where $g_{1}$ is defined by $g_{1}(x)=\int_{a}^{x} h(t) d t$, it follows that $f \mid P=$ $g \mid P$, and this completes the proof for the case where $f \in C^{1 \oplus 1}$.

The case where $f \in D^{2}$ follows easily. 
Example 10. There exists a $C^{1 \oplus 1}$ function $f$ such that for every $D^{2} g$ the set $\{x: f(x)=g(x)\}$ is nowhere dense.

Proof. To obtain this example, just take the indefinite integral of the function of Example 4.

\section{REFERENCES}

1. S. Agronski, A. M. Bruckner, M. Laczkovich, and D. Preiss, Convexity conditions and intersections with smooth functions, Trans. Amer. Math. Soc. 289 (1985), 659-677.

2. H. Federer, Surface area. I, II, Trans. Amer. Math. Soc. 55 (1944), 420-456.

3. _ Geometric measure theory, Springer-Verlag, New York, 1969.

4. K. Kuratowski, Topology, Vol. I, Academic Press, New York and London, 1966.

5. M. Laczkovich, Differentiable restrictions of real functions, Acta Math. Hungar. 44 (1984), 355-360.

6. S. Minakshisundaram, On the roots of a continuous non-differentiable function, J. Indian Math. Soc. 4 (1940), 31-33.

7. A. Olevskii, Ulam-Zahorski problem on free interpolation by smooth functions, Trans. Amer. Math. Soc. 342 (1994), 713-727 (see summary in Real Analysis Exchange 16 (1990/91), 363-372).

8. H. Whitney, Analytic extensions of differentiable functions defined in closed sets, Trans. Amer. Math. Soc. 36 (1934), 63-89.

19. __ On totally differentiable and smooth functions, Pacific J. Math. 1 (1951), 143-159.

Department of Mathematics, Auburn University, Alabama 36949-5310 